\title{
Participación comunitaria en el control de las parasitosis intestinales en una localidad rural de Argentina
}

\author{
Betina C. Pezzani, ${ }^{1}$ Marta C. Minvielle,$^{1}$ María L. Ciarmela, ${ }^{1}$ \\ María C. Apezteguía ${ }^{2}$ y Juan A. Basualdo ${ }^{1}$
}

Forma de citar Pezzani BC, Minvielle MC, Ciarmela ML, Apezteguía MC, Basualdo JA. Participación comunitaria en el control de las parasitosis intestinales en una localidad rural de Argentina. Rev Panam Salud Publica. 2009;26(6):471-7.

RESUMEN Objetivos. Diseñar, implementar y evaluar un plan complejo de acciones dirigido a reducir las parasitosis en una localidad rural de la provincia de Buenos Aires, Argentina, con la participación de la comunidad.

Métodos. El trabajo se realizó en General Mansilla, provincia de Buenos Aires, Argentina, en tres etapas. 1) Se evaluó la situación epidemiológica inicial de las parasitosis intestinales en la comunidad mediante análisis coproparasitológico seriado y de escobillado anal en una muestra de 522 personas. 2) Se implementaron dos intervenciones: el tratamiento farmacológico de las personas parasitadas y la educación sanitaria de la población con la participación activa de divulgadores locales. 3) El tratamiento antiparasitario se evaluó mediante análisis coproparasitológico de seguimiento de todas las personas tratadas; la intervención educativa se evaluó mediante una encuesta y análisis coproparasitológico a personas que solo habían participado en el plan de educación sanitaria.

Resultados. La frecuencia de parasitosis intestinal fue de 58,2\%; del total, 43,9\% por protozoos y 35,2\% por helmintos. Los patógenos más frecuentes fueron Enterobius vermicularis, Blastocystis hominis y Giardia lamblia. El tratamiento antiparasitario redujo la parasitosis intestinal a 15,1\% ( $\mathrm{P}<0,001)$, más eficaz en helmintos que en protozoos. La parasitosis intestinal disminuyó después de la intervención educativa sanitaria, tanto en sentido general (de 58,2\% a 47,9\%; $\mathrm{P}=0,019$ ) como por helmintos (de 35,2\% a 20,3\%; $\mathrm{P}<0,001$ ) y se mejoraron significativamente los hábitos higiénicos.

Conclusiones. El tratamiento parasitológico y la intervención educacional mediante divulgadores locales permitieron reducir las parasitosis en la comunidad estudiada, especialmente las provocadas por helmintos. Se recomienda extender esta experiencia a otras comunidades rurales y ampliarla con intervenciones adicionales dirigidas a cortar otras vías de transmisión, como el agua y los alimentos.

Palabras clave Parasitosis intestinales; educación en salud; auxiliares de salud comunitaria; Argentina.

La parasitosis intestinal es un grave problema de salud en el mundo, espe-

\footnotetext{
Cátedra de Microbiología y Parasitología, Facultad de Ciencias Médicas, Universidad Nacional de La Plata, La Plata, Argentina. Dirección postal: Cátedra de Microbiología y Parasitología, Facultad de Ciencias Médicas, Universidad Nacional de La Plata, 60 y 120, La Plata 1900, Argentina. Correo electrónico: jabasua@atlas.med. unlp.edu.ar

2 Comisión de Investigaciones Científicas de la Provincia de Buenos Aires, La Plata, Provincia de Buenos Aires, Argentina.
}

cialmente en los países de menor desarrollo económico y en las zonas pobres y rurales de la mayoría de los países del mundo. De hecho, la incidencia y la prevalencia de parasitosis intestinales se han tomado como indicadores del estado de salud de la población en distintos contextos (1).

Las elevadas tasas de infestación por parásitos intestinales en países latinoamericanos son un reflejo de la situación en la que viven sus habitantes, en ocasiones persistentemente expuestos a un entorno contaminado con parásitos, además de las deficiencias en los hábitos de higiene $(2,3)$. La transmisión de parásitos se ve favorecida por la contaminación fecal del agua de consumo, el suelo y los alimentos y por factores socioculturales, como el nivel socioeconómico y educacional y las prácticas de higiene de la población $(4,5)$. 
Tanto las tasas de infestación por parásitos intestinales como el espectro de especies predominantes varían considerablemente de una localidad a otra. En una investigación realizada en escolares mexicanos, las especies más prevalentes fueron Entamoeba histolytica (10,5\%), Giardia lamblia $(8,1 \%)$ y Ascaris lumbricoides $(6,0 \%)$ y se concluyó que las altas tasas de infestación encontradas en los municipios de elevada marginalidad estaban relacionadas con las condiciones de pobreza y la falta de servicios básicos (6). En Colombia se ha encontrado una alta prevalencia de geohelmintosis en menores de 15 años de La Virgen, una zona rural del municipio de Quipile, departamento de Cundinamarca, donde las viviendas estaban en mal estado y carecían de servicios adecuados de agua potable y eliminación de excretas. Sin embargo, a pesar de que las condiciones socioeconómicas se mantuvieron sin cambios, la prevalencia de infestación con los principales parásitos se redujo notablemente en 10 años (A. lumbricoides de $36,2 \%$ a $27,7 \%$, Trichuris trichiura de $37,1 \%$ a $21,2 \%$ y Uncinaria sp. de $19,6 \%$ a $7,1 \%$ ), posiblemente debido a la educación y la conciencia colectiva adquiridas por esta comunidad durante el prolongado seguimiento realizado a esta población, lo que puede haber mejorado el conocimiento, las prácticas y los hábitos sanitarios de sus pobladores (7). Esto indica que poblaciones con grandes carencias económicas son susceptibles a intervenciones comunitarias (quimioterapia, educación y saneamiento) dirigidas a controlar el grave problema del parasitismo intestinal.

En Argentina, estudios descriptivos han informado de prevalencias de parasitosis por encima de $80,0 \%$ en algunas localidades del norte y el sur del país (8-11), mientras que en la zona central se registran porcentajes cercanos a $45,0 \%$ (12).

Estudios realizados en General Mansilla, provincia de Buenos Aires, Argentina, demostraron que algunas variables (hogar de cartón, chapa o madera; piso de tierra; bomba de agua; llave de agua fuera del hogar o pública; y presencia de pozo ciego o letrina) se relacionaron con la presencia de parásitos intestinales (5). En esa comunidad se observó una asociación significativa de la infestación del suelo y el anegamiento frecuente del perímetro domiciliario, con la frecuencia de toxocariosis en la población (13). El estudio de factores de riesgo de infestación como estos puede contribuir a elaborar políticas y diseñar intervenciones eficaces dirigidas a cambiar la situación sanitaria local y evitar la diseminación de las enfermedades parasitarias.

Sin embargo, el control de las parasitosis es una tarea compleja. Durante más de dos décadas se han realizado esfuerzos para reducir las helmintosis en América Latina. Entre esos esfuerzos se pueden mencionar las intervenciones llevadas a cabo en Ecuador y México en niños en edad escolar y preescolar (14, 15), los programas y las campañas de suministro masivo de antihelmínticos de amplio espectro coordinados por la Organización Mundial de la Salud (OMS) en comunidades con elevada prevalencia de geohelmintos $(16,17)$ y el programa de tratamiento masivo y reiterado planteado en Argentina para reducir la prevalencia y la carga parasitaria de $A$. lumbricoides (18).

En Argentina, el Programa Nacional de Tratamientos Masivos Antiparasitarios, impulsado por el Ministerio de Salud de la Nación (19), está dirigido solamente a los niños en edad preescolar (2-5 años) y escolar (5-14 años) y se ejecuta mediante agentes sanitarios, médicos comunitarios y promotores de salud que entregan los medicamentos a los responsables de los niños directamente en sus domicilios (no se indica a menores de 2 años porque no forman parte de este programa). El tratamiento completo consiste en cuatro dosis sucesivas de $500 \mathrm{mg}$ de mebendazol cada seis meses. El Ministerio de Salud escogió este antihelmíntico por su bajo costo, su eficacia y los mínimos efectos secundarios que provoca con las dosis utilizadas: dolor abdominal y diarreas leves y transitorios y migraciones ocasionales de A. lumbricoides (eliminación por la nariz o la boca). Este es el único medicamento antihelmíntico que reciben los centros de salud que participan en ese programa.

No obstante, la aplicación del tratamiento farmacológico no es suficiente para reducir la infestación con helmintos si no se cambian los hábitos higiénicos y sanitarios inadecuados que pueden llevar a una reinfestación. Por ello se deben implementar intervenciones eficaces de comunicación dirigidas a los sectores de la población más vulnerables (20). En estas intervenciones deben participar los planificadores, el equipo de salud y la comunidad. Entre las metodologías par- ticipativas dirigidas a la educación sanitaria se destacan la denominada Transformación Participativa de la Higiene y el Saneamiento (PHAST), promovida por la OMS (21), y la estrategia SARAR (22) - basada en elevar los atributos y las capacidades esenciales para dinamizar el cambio de conducta: autoestima, fuerza asociativa, inventiva, planeación de acciones y responsabilidad- que PROANDE ha aplicado en comunidades andinas de Perú (23).

Otro pilar que debe formar parte de cualquier intervención es el mejoramiento de la infraestructura sanitaria de la población. El saneamiento es la principal medida para eliminar las geohelmintosis, aunque para ser eficaz debe abarcar una gran parte de la población (24). Sin embargo, estas medidas tienen un elevado costo y no pueden aplicarse cuando los recursos son limitados. Además, se debe implementar durante años o incluso décadas para alcanzar los resultados esperados (25).

El objetivo del presente trabajo fue diseñar, implementar y evaluar un plan complejo de acciones dirigido a reducir las parasitosis en una localidad rural de la provincia de Buenos Aires, Argentina, con la participación de la comunidad.

\section{MATERIALES Y MÉTODOS}

El trabajo se llevó a cabo en la localidad General Mansilla en tres etapas: 1) caracterización de la situación epidemiológica inicial de las parasitosis intestinales en la comunidad estudiada; 2) implementación de dos intervenciones - una terapéutica y una educativapara el control de las parasitosis; y 3) evaluación de las intervenciones implementadas.

En General Mansilla —localidad eminentemente ganadera del partido Magdalena, ubicada al noreste de la provincia de Buenos Aires, Argentina- viven 1684 personas (hombres: 49,3\%; mujeres: 50,7\%), según el Censo Nacional de Población, Hogares y Viviendas de 2001 (26). La población urbana se abastece de agua del acuífero Puelche, almacenada y clorada por una cooperativa local, mientras que la rural consume agua de pozos individuales de $15-20 \mathrm{~m}$ de profundidad. Si bien en el casco urbano $70,0 \%$ de los habitantes se sirve de cloacas para desechar sus aguas servidas, el resto utiliza letrinas o pozos ciegos individuales. Esta localidad cuenta con un centro de 
salud que brinda servicios de atención primaria, mientras el hospital municipal más cercano se encuentra a $28 \mathrm{~km}$, en la ciudad cabecera del partido.

\section{Caracterización de la situación epidemiológica inicial}

Para el diagnóstico inicial de las parasitosis intestinales se seleccionó una muestra de 522 personas -263 $(50,4 \%)$ varones y $259(49,6 \%)$ mujeres- que asistieron consecutivamente al centro de salud local entre agosto y diciembre de 2004 con alguna molestia (síndromes respiratorios, digestivos, traumáticos, etc.) que no requirieron derivación de urgencia a un hospital, o sin síntomas (personas que iban como acompañantes, en busca de turnos o por controles periódicos, vacunas, consultas odontológicas y entrevistas con psicólogos, entre otros). De la muestra, 381 (72,9\%) eran niños (14 años o menos).

A cada participante se le prescribió un análisis coproparasitológico seriado (27) y de escobillado anal (28) y se le aplicó una encuesta sociocultural que recababa información sobre la localización de la vivienda (rural o urbana), su material de construcción (cartón, chapa, madera, fibrocemento o mampostería), la vía de eliminación de residuos (a cielo abierto, se quema, se entierra o recolección municipal), tipo de baño (instalado, letrina o no posee), sistema de eliminación de las excretas (red cloacal, pozo, letrina o a cielo abierto), grado de hacinamiento (más o menos de tres personas por habitación), si la vivienda sufre anegamientos (sí o no) y si tiene mascota (sí o no).

Para el análisis coproparasitológico se tomó diariamente durante 5 días consecutivos una porción de materia fecal emitida espontáneamente, en un frasco descartable con tapa de rosca que contenía formol al 5\%. Estas nuestras se procesaron mediante la técnica de Telemann modificada, recomendada por el Ministerio de Salud de la Nación (29). Brevemente: se maceraron $10 \mathrm{~g}$ de las muestras homogeneizadas en $15 \mathrm{~mL}$ de solución de Telemann (preparada con $5 \mathrm{~g}$ de cloruro de sodio, $50 \mathrm{~mL}$ de formol al $40 \%$ y $950 \mathrm{~mL}$ de agua destilada). Después de filtrar por una capa doble de gasa se agregó $1 \mathrm{~mL}$ de éter y se centrifugó a 1000 gravedades durante 3 minutos. La detección microscópica de trofozoítos y quistes de protozoos, así como de huevos y larvas de helmintos, se rea- lizó con aumentos de 100, 450 y 1000 veces en seis frotis de cada muestra, tres tratados con una gota de lugol y tres con colorante de Kinyoun (30).

Para el escobillado anal, los participantes pasaron una gasa húmeda por los bordes del orificio anal al despertarse por la mañana y las colocaron en un frasco descartable con tapa de rosca que contenía formol al 5\%. Una vez en el laboratorio, después de cortar y homogeneizar las gasas con el formol del frasco, se concentró el contenido mediante centrifugación a 1000 gravedades durante 5 minutos y se observó al microscopio el pellet obtenido con aumentos de $100 \mathrm{y}$ 450 veces (31).

Las muestras se recibieron semanalmente en el centro de salud y se procesaron en el Laboratorio de Parasitología de la Facultad de Ciencias Médicas de la Universidad Nacional de La Plata, Argentina. Los informes de los resultados se entregaron la semana siguiente a la recepción de la muestra.

\section{Implementación de intervenciones para el control de las parasitosis}

Las medidas de control consistieron en la aplicación de dos intervenciones: el tratamiento farmacológico de las personas parasitadas y la educación sanitaria de la población con la participación activa de divulgadores locales.

El tratamiento se realizó selectivamente a las personas que concurrían al centro de salud a retirar sus informes parasitológicos y estos eran positivos. Los médicos del centro aplicaron el tratamiento antiparasitario en la propia institución, según las recomendaciones del Programa
REMEDIAR (19) y Atias (1) (cuadro 1). No se realizó atención domiciliaria.

La intervención de educación sanitaria se implementó entre marzo y noviembre de 2005 y estuvo a cargo de alumnos seleccionados del ciclo polimodal (15 a 17 años) de la Escuela de Enseñanza Agraria No. 801 de General Mansilla. Se seleccionaron como divulgadores locales a los alumnos que manifestaron interés por el proyecto, motivados por la posibilidad de introducir cambios para promover el bienestar de su comunidad. Los alumnos se entrenaron y evaluaron en temas de morfología y biología de los parásitos, morbilidad y mortalidad por enfermedades parasitarias, mecanismos y vías de transmisión, ciclos evolutivos, métodos de diagnóstico y medidas de prevención. Las parasitosis que se abordaron fueron las prevalentes en la comunidad.

Los divulgadores locales evaluaron los hábitos saludables y no saludables (de riesgo) de la comunidad, identificaron las fuentes y las vías de transmisión de las parasitosis, así como los reservorios y las barreras que pudieran reducir la diseminación parasitaria. Con los resultados de este análisis se identificaron las modificaciones en los hábitos y costumbres necesarios para generar un cambio en la situación epidemiológica y se prepararon los mensajes fundamentales para los encuentros educativos con la comunidad.

Para estos encuentros, realizados en las escuelas de la localidad, las autoridades de cada institución convocaron a los alumnos, sus padres, el personal de la escuela (incluidos los responsables de la limpieza) y a todas las personas interesadas en asistir. En las escuelas del casco

CUADRO 1. Tratamientos aplicados durante la intervención terapéutica a las personas parasitadas, General Mansilla, Argentina, 2004

\begin{tabular}{|c|c|c|}
\hline Parásito & Medicamento & Tratamiento \\
\hline Giardia lamblia ${ }^{a}$ & Metronidazol & $\begin{array}{l}\text { Niños: } 15 \mathrm{mg} / \mathrm{kg} / \text { día en } 3 \text { dosis/día, } 7 \text { días } \\
\text { Adultos: } 250 \mathrm{mg} \text { cada } 8 \mathrm{~h}, 7 \text { días }\end{array}$ \\
\hline Blastocystis hominis a & Metronidazol & $\begin{array}{l}\text { Niños: } 35-50 \mathrm{mg} / \mathrm{kg} / \mathrm{día} \text { en } 3 \text { dosis/día, } 7 \text { días } \\
\text { Adultos: } 250 \mathrm{mg} \text { cada } 8 \text { h, } 7 \text { días }\end{array}$ \\
\hline Ascaris lumbricoides ${ }^{a}$ & Mebendazol & $\begin{array}{l}\text { Niños y adultos: } 100 \text { mg cada } 12 \text { h, } 3 \text { días; repetir a los } \\
14 \text { días }\end{array}$ \\
\hline Enterobius vermicularis ${ }^{a}$ & Mebendazol & Niños y adultos: 100 mg una vez; repetir a los 14 días \\
\hline Trichuris trichiura ${ }^{a}$ & Mebendazol & Niños y adultos: $100 \mathrm{mg} / \mathrm{día}, 3$ días \\
\hline Strongyloides stercoralis ${ }^{b}$ & Tiabendazol & $\begin{array}{l}\text { Niños: } 50 \mathrm{mg} / \mathrm{kg} \text {, en } 2 \text { dosis/día, } 2 \text { días } \\
\text { Adultos: } 1500 \mathrm{mg}, 2 \text { veces/día, } 2 \text { días }\end{array}$ \\
\hline Hymenolepis nana & Praziquantel & $\begin{array}{l}\text { Niños: } 20-25 \mathrm{mg} / \mathrm{kg} \text { una vez } \\
\text { Adultos: } 1500 \mathrm{mg} \text { una vez }\end{array}$ \\
\hline
\end{tabular}

a Según las recomendaciones del Programa PROAPS/REMEDIAR (19).

b Según recomendaciones de Atías (1). 
urbano, los encuentros se realizaron por separado para cada grado de la educación general básica (de $1 .^{\circ}$ a $6 .^{\circ}$ grados) y el jardín de infantes. En las escuelas rurales se realizaron encuentros conjuntos, debido al escaso número de alumnos en cada grado y a que estos escolares desarrollan sus actividades conjuntamente y con un solo maestro. Los encuentros estuvieron a cargo de los divulgadores locales bajo la supervisión del equipo de docentes de la Universidad Nacional de La Plata.

Se realizaron tres encuentros con cada grupo, para un total de 39 encuentros. Se utilizaron láminas con dibujos de los ciclos biológicos de los parásitos presentes en la comunidad y proyecciones de filminas que resaltaban las conductas que la comunidad podía implementar para evitar la transmisión de los parásitos. Participaron todas las escuelas urbanas (una de educación general básica y un jardín de infantes) y los seis establecimientos rurales de educación general básica. En cada encuentro se estableció un intercambio fluido con los padres, los maestros y los escolares y se hizo hincapié en la importancia de lavarse las manos, lavar las verduras y las frutas y mantener buenos hábitos de higiene personal y comunitaria, ya que actúan como barreras sanitarias.

\section{Evaluación de las intervenciones implementadas}

Para medir la eficacia del tratamiento antiparasitario aplicado se repitió el estudio parasitológico seriado (coproparasitológico y escobillado anal) cuatro semanas después de terminado el tratamiento. Se compararon las frecuencias de parasitosis antes y después del tratamiento mediante la prueba de Mc Nemar.

Para evaluar los resultados de la intervención educativa se realizaron análisis parasitológicos (coproparasitológico y escobillado anal) a una nueva muestra de 167 personas $-75(44,9 \%)$ varones y $92(55,1 \%)$ mujeres-, de los cuales 121 $(72,5 \%)$ eran niños y $46(27,5 \%)$ adultos que acudieron al centro de salud entre el 1 de octubre y el 1 de diciembre de 2006, independientemente de la presencia de síntomas. Los criterios de inclusión fueron no haber tomado parte en la primera etapa de esta investigación y haber participado en la intervención de educación sanitaria. Las personas mayores de 10 años completaron de manera anónima un breve cuestionario autoadministrado que indagaba sobre posibles cambios de conducta (onicofagia, lavado de manos, posibles vías de infección con materia fecal, entre otras). También se realizó una breve encuesta a las autoridades de los ocho establecimientos educativos participantes sobre la frecuencia del aseo de los baños. Los cuestionarios se validaron mediante una prueba piloto previa con 30 personas de la localidad.

\section{Análisis de los resultados}

Se calcularon las frecuencias generales y específicas por parasitosis, sexo y grupo de edad, tanto antes como después de la intervención, y se compararon los valores mediante las pruebas de la ji al cuadrado y de Fisher. Los análisis estadísticos se realizaron con el programa informático SPSS ${ }^{\circledR}$ versión 11.5.

Todos los adultos y los responsables de los menores participantes firmaron una declaración de consentimiento informado. Este protocolo de investigación fue aprobado por la Comisión de Investigaciones Científicas y el Consejo Superior de la Universidad Nacional de La Plata, y forma parte del Programa de Incentivo a los Docentes Investigadores.

\section{RESULTADOS}

La frecuencia general de parasitosis intestinales fue de 58,2\%; del total, 43,9\% por protozoos y $35,2 \%$ por helmintos. No se encontraron diferencias estadísticamente significativas entre las variables estudiadas según el sexo o los grupos de edad (cuadro 2). Sin embargo, en un análisis secundario en que se analizaron los niños según cuatro grupos de edad que correspondían a diferentes niveles de socialización, se encontró que el grupo de 6 a 8 años presentó una mayor frecuencia de parásitos intestinales $(76,5 \%)$ que los niños de 0 a 2, de 3 a 5 y de 9 a 14 años $(P<0,001)$.

Se detectaron parásitos intestinales patógenos y comensales. Los patógenos más frecuentes fueron Enterobius vermicularis, Blastocystis hominis y Giardia lamblia y entre los considerados no patógenos, los más frecuentes fueron las denominadas amebas comensales: Entamoeba polecki, Entamoeba hartmanni, Endolimax nana, Iodamoeba bütschlii, Retortamonas intestinalis, Enteromona hominis y Entamoeba coli (cuadro 3).

\section{Resultados del tratamiento antiparasitario}

De las 304 personas tratadas, se recibieron y procesaron las muestras de seguimiento de 271 (208 niños y 63 adultos; 149 varones y 122 mujeres). La frecuencia de parasitosis intestinales disminuyó con respecto a lo observado antes del tratamiento, tanto en general $(58,2 \%$ frente a $15,1 \%, P<0,001)$ como por protozoos (de $43,9 \%$ a 9,9\%; $P<0,001$ ) y helmintos (de $35,2 \%$ a $8,1 \% ; P<0,001$ ) (cuadro 2).

En general, el tratamiento fue más eficaz en helmintos que en protozoos. Se encontraron reducciones de $100 \%$ en A. lumbricoides, T. trichiura, Strongyloides stercoralis, Hymenolepis nana, Isospora belli, E. nana, coccidios y Cryptosporidium spp., así como de $91,4 \%$ en B. hominis, $91,2 \%$ en G. lamblia y $88,8 \%$ en E. vermicularis (sin contar los casos de reinfestación).

CUADRO 2. Parasitosis antes y después de las dos intervenciones realizadas en General Mansilla, Argentina, 2004-2006

\begin{tabular}{|c|c|c|c|c|c|c|}
\hline \multirow[b]{2}{*}{ Variable } & \multicolumn{2}{|c|}{$\begin{array}{l}\text { Antes de las } \\
\text { intervenciones } \\
\quad(n=522)\end{array}$} & \multicolumn{2}{|c|}{$\begin{array}{c}\text { Después de la } \\
\text { intervención farmacológica } \\
(n=271)\end{array}$} & \multicolumn{2}{|c|}{$\begin{array}{c}\text { Después de la } \\
\text { intervención educativa } \\
(n=167)\end{array}$} \\
\hline & Casos & $\%$ & Casos & $\%$ & Casos & $\%$ \\
\hline Total & 304 & 58,2 & 41 & $15,1^{\mathrm{a}}$ & 80 & $47,9^{b}$ \\
\hline \multicolumn{7}{|l|}{ Parásitos } \\
\hline Protozoos & 229 & 43,9 & 27 & $9,9^{a}$ & 65 & 38,9 \\
\hline Helmintos & 184 & 35,2 & 22 & $8,1^{a}$ & 34 & $20,3^{a}$ \\
\hline \multicolumn{7}{|l|}{ Sexo } \\
\hline Mujeres & 141 & 54,4 & 15 & 12,3 & 41 & 44,6 \\
\hline Hombres & 163 & 62,0 & 26 & 17,4 & 39 & 52,0 \\
\hline \multicolumn{7}{|l|}{ Edad, años } \\
\hline$\leq 14$ & 231 & 60,6 & 36 & 17,3 & 62 & 51,9 \\
\hline$>14$ & 73 & 51,8 & 5 & 7,9 & 46 & 39,1 \\
\hline
\end{tabular}

a Diferencia significativa $P<0,001$ con respecto a antes de la intervención.

${ }^{b}$ Diferencia significativa $P=0,019$ con respecto a antes de la intervención. 
CUADRO 3. Parasitosis intestinales encontradas antes y después de las dos intervenciones realizadas, según la especie, General Mansilla, Argentina, 2004-2006

\begin{tabular}{|c|c|c|c|c|c|c|c|c|}
\hline \multirow[b]{2}{*}{ Especie de parásito } & \multicolumn{2}{|c|}{$\begin{array}{c}\text { Antes de las } \\
\text { intervenciones } \\
\quad(n=522)\end{array}$} & \multicolumn{3}{|c|}{$\begin{array}{l}\text { Después de la } \\
\text { intervención } \\
\text { farmacológica } \\
\quad(n=271)\end{array}$} & \multicolumn{3}{|c|}{$\begin{array}{c}\text { Después de la } \\
\text { intervención educativa } \\
(n=167)\end{array}$} \\
\hline & Casos & $\%$ & Casos & $\%$ & $P^{b}$ & Casos & $\%$ & $P^{b}$ \\
\hline \multicolumn{9}{|l|}{ Protozoos } \\
\hline Blastocystis hominis & 143 & 27,4 & 20 & 7,4 & $<0,001$ & 42 & 25,1 & 0,638 \\
\hline Giardia lamblia & 38 & 7,3 & 7 & 2,6 & 0,010 & 20 & 12,0 & 0,057 \\
\hline Entamoeba coli & 57 & 10,9 & 7 & 2,6 & $<0,001$ & 14 & 8,4 & 0,428 \\
\hline Endolimax nana & 18 & 3,4 & 0 & 0 & 0,004 & 1 & 0,6 & 0,050 \\
\hline Amebas & 64 & 12,3 & 4 & 1,5 & $<0,001$ & 11 & 6,6 & 0,040 \\
\hline Coccidios & 4 & 0,8 & 0 & 0 & 0,359 & 2 & 1,2 & 0,965 \\
\hline Chilomastix mesnili & 5 & 1,0 & 1 & 0,4 & 0,634 & 0 & 0 & 0,455 \\
\hline Cryptosporidium & 1 & 0,2 & 0 & 0 & 0,470 & 0 & 0 & 0,571 \\
\hline Isospora belli & 1 & 0,2 & 0 & 0 & 0,470 & 0 & 0 & 0,571 \\
\hline \multicolumn{9}{|l|}{ Helmintos } \\
\hline Ascaris lumbricoides & 19 & 3,6 & 0 & 0 & 0,003 & 2 & 1,2 & 0,180 \\
\hline Hymenolepis nana & 4 & 0,8 & 0 & 0 & 0,359 & 4 & 2,4 & 0,180 \\
\hline Enterobius vermicularis & 171 & 32,8 & 22 & 8,1 & $<0,001$ & 30 & 18,0 & $<0,001$ \\
\hline Strongyloides stercoralis & 1 & 0,2 & 0 & 0 & 0,470 & 1 & 0,6 & 0,979 \\
\hline Trichuris trichiura & 9 & 1,7 & 0 & 0 & 0,470 & 1 & 0,6 & 0,492 \\
\hline
\end{tabular}

a Se incluyen los casos de reinfestación.

${ }^{b}$ Con respecto a antes de las intervenciones según la prueba de la ji al cuadrado. Nivel de significación $P \leq 0,05$.

\section{Resultados de la intervención educativa}

La frecuencia general de parasitosis intestinal disminuyó después de la intervención educativa sanitaria, tanto en sentido general (de $58,2 \%$ a $47,9 \% ; P=0,019$ ) como por helmintos (de $35,2 \%$ a $20,3 \%$; $P<0,001)$. Aunque se observó también una reducción en la frecuencia de protozoosis (de $43,9 \%$ a $38,9 \%$ ), esta no fue significativa $(P>0,05)$. Las frecuencias de parásitos intestinales según el género y el grupo de edad también disminuyeron después de la intervención educativa, aunque sin alcanzar el nivel de significación.

Con relación a las especies de parásitos encontradas, solamente se redujo significativamente la frecuencia de amebas (de $12,3 \%$ a $6,6 \% ; P=0,040$ ) y E. vermicularis (de $32,8 \%$ a $18,0 \% ; P<0,001$ ).

En los niños, la frecuencia de parasitosis intestinal descendió, pero ese descenso fue estadísticamente significativo solamente en los grupos de 6 a 8 años (de $76,5 \%$ a $56,7 \% ; P=0,039)$ y de 9 a 14 años (de $66,1 \%$ a $47,2 \% ; P=0,042$ ).

\section{Resultado de las encuestas}

Según los resultados de las encuestas, antes del encuentro educativo $70,7 \%$ de los participantes se comía las uñas (onicofagia), 90,2\% se llevaba juguetes o lapiceros a la boca, $50,0 \%$ no se lavaba las manos antes de comer y 59,8\% no lo hacía después de ir al baño. Después del encuentro educativo, estos porcentajes se redujeron significativamente $(P<0,001)$ a $9,7 \%, 9,7 \%, 19,5 \%$ y 19,5\%. También aumentó significativamente el número de participantes que consideraba la materia fecal de mascotas o animales de granja como fuente de parásitos (de 20,7\% a $100 \%$; $P<0,001)$. Respecto a las modificaciones en las instituciones educativas, se logró que la limpieza de los baños al terminar cada turno escolar ascendiera de $50,0 \%$ a $100 \%(P<0,001)$.

\section{DISCUSIÓN}

Con frecuencia, las intervenciones implementadas como parte de los planes de salud pública parten de una concepción vertical, en la que el agente de salud se encuentra en un plano distante y es percibido como una persona que impone ideas que la comunidad no comparte. Con la participación de jóvenes de la comunidad como divulgadores y el empleo de un lenguaje común se puede lograr una mayor motivación para cambiar los hábitos de higiene. El empleo de materiales visuales adaptados a la cultura local y enfocados a todos los grupos de edad permitió involucrar eficazmente a la población y facilitó que más personas -tanto escolares como sus familiaresparticiparan en los encuentros realizados en los establecimientos educativos de la localidad. La elección de la escuela como lugar de encuentro permitió aumentar la asistencia, pues la distancia desde los hogares no constituyó una barrera.

En la primera etapa del proyecto se determinó que los parásitos patógenos más frecuentes eran E. vermicularis, B. hominis y G. lamblia. La transmisión de $E$. vermicularis está directamente relacionada con la onicofagia y la incorrecta higiene individual y familiar (32), mientras que los dos restantes se transmiten directa o indirectamente por el agua contaminada $(33,34) ;$ G. lamblia también se puede transmitir por los animales (35).

A diferencia de estudios realizados en otras poblaciones argentinas $(10,18)$, en la presente investigación se encontraron relativamente muy pocos casos de geohelmintosis. Esto puede deberse a la baja densidad poblacional en General Mantilla, lo que puede haber generado una menor presión de infestación por el menor número de exposiciones a las formas infectivas de los geohelmintos (1). Además, se debe tener en cuenta que $70,0 \%$ de la población del casco urbano contaba con servicio cloacal, lo que contribuye a disminuir el riesgo de infestación con este tipo de helmintos.

La frecuencia de parasitosis intestinal infantil fue mayor en los niños de 6 a 8 años — coincidente con lo informado por otros autores $(36,37)$ - mientras que en el grupo de 0 a 2 años se observó la menor frecuencia. Esto puede deberse a que en el ámbito rural, los niños menores permanecen en sus hogares y tienen menos contacto con las fuentes de infección, mientras que a partir de los 6 años ingresan al sistema educativo obligatorio, donde se presentan las condiciones propicias para la transmisión directa de los parásitos.

El tratamiento antiparasitario tuvo una elevada eficacia de más de $85 \%$ para todos los parásitos tratados. Los patógenos que persistieron fueron G. lamblia, $B$. hominis y, como único helminto, E. vermicularis. Esto puede explicarse por los cortos períodos prepatentes ${ }^{2}$ de estas especies - menores que el tiempo transcurrido entre el tratamiento y el análisis de control-, por lo que algunos casos podrían ser reinfestaciones.

En cuanto a la frecuencia general de parasitosis intestinal después de la intervención educativa, si bien persistieron las mismas especies de parásitos, se ob-

\footnotetext{
2 Etapa de la infección parasitaria comprendida entre el momento de la infección y la aparición de síntomas o la presencia detectable del parásito.
} 
servó una disminución significativa en las frecuencias y una modificación en el orden de importancia: después de la intervención educativa, E. vermicularis dejó de ser la especie predominante para pasar a la segunda posición por debajo de $B$. hominis. Los geohelmintos que presentaban una baja frecuencia antes de la intervención disminuyeron aún más después de ella; el impacto de la intervención educativa fue menor en el control de los protozoos. Esto demuestra la eficacia de las medidas implementadas para el control de la parasitosis en general y las helmintosis en particular.

La disminución en la frecuencia de $E$. vermicularis —favorecida por la presencia de formas viables en los entornos familiar y escolar - podría deberse a la aplicación de las medidas de higiene, principalmente individuales y familiares, promovidas por los divulgadores en la comunidad durante la intervención de educación sanitaria, basada en mensajes claros y precisos.

Los cambios en los hábitos y conductas personales y familiares tuvieron un menor efecto en la transmisión de B. hominis y G. lamblia, posiblemente debido a la existencia de otras vías de transmisión (agua o alimentos). Se sugirió a la comunidad filtrar el agua de consumo mediante siste- mas de filtración doble, con prefiltro de sedimentos y filtro de carbón activado en bloque, ya que la cloración no garantiza la inactivación de los quistes (38).

El grupo de niños de 6 a 8 años se mantuvo como el de mayor frecuencia general de parasitosis intestinal en la población infantil antes y después de la intervención. No obstante, la frecuencia general descendió en todos los grupos de edad después de la intervención y esta reducción fue significativa en los grupos de 6 a 8 y de 9 a 14. Esto podría explicarse por la mayor receptividad de estos niños a las explicaciones y por la posibilidad de que ellos mismos sean generadores de conductas saludables.

Lograr cambios en los hábitos de higiene de la población es una tarea principal de la educación sanitaria, por lo que este componente no puede permanecer marginado por más tiempo de los proyectos e intervenciones llevados a cabo en Argentina.

Las limitaciones más importantes que enfrentó este trabajo fueron las distancias entre los puntos de intervención en las zonas rurales y las características del terreno, que limitaron las posibilidades operativas para realizar un seguimiento personal. No obstante, después del trata- miento se logró estudiar a $89,1 \%$ de las personas evaluadas antes de las intervenciones, lo que reflejó el interés de la comunidad en el problema planteado.

El tratamiento parasitológico individual y selectivo de las personas parasitadas en la comunidad estudiada y la intervención de educación sanitaria realizada con participación de divulgadores locales permitieron reducir las parasitosis, especialmente las provocadas por helmintos. Los cambios en los hábitos y las conductas personales y familiares tuvieron un menor efecto sobre las protozoosis. Se recomienda extender esta experiencia a otras comunidades rurales y ampliarla con intervenciones adicionales dirigidas específicamente a cortar otras vías de transmisión, como el agua y los alimentos.

Agradecimientos. Se agradece a Roberto Zungri por facilitar el ingreso a la comunidad General Mansilla. Este estudio recibió financiamiento de la Agencia Nacional de Promoción Científica y Técnica, la Fundación Alberto J. Roemmers y la Universidad Nacional de La Plata, todas de Argentina. Este proyecto fue declarado de interés municipal por el partido de Magdalena, provincia de Buenos Aires, Argentina.

\section{REFERENCIAS}

1. Atías A. Parasitología médica. Santiago: Mediterráneo; 1999.

2. Iannacone J, Benites MJ, Chirinos L. Prevalencia de infección por parásitos intestinales en escolares de primaria de Santiago de Surco, Lima, Perú. Parasitol Latinoam. 2006;61: 54-62.

3. Devera R, Angulo V, Amaro E, Finali M, Franceshi G, Blanco Y, et al. Parásitos intestinales en habitantes de una comunidad rural del Estado Bolívar, Venezuela. Rev Biomed. 2006;17:259-68.

4. Cifuentes E, Blumenthal U, Ruiz-Palacios G, Bennett S, Pesaey A. Escenario epidemiológico del uso agrícola del agua residual: el Valle del Mezquital, México. Salud Publica Mex. 1994;36:3-9.

5. Mason P, Patterson B. Epidemiology of $\mathrm{Hy}$ menolepis nana infections in primary school children in urban and rural communities in Zimbawe. J Parasitol. 1994;80:245-50.

6. Guerrero Hernández MT, Hernández Molinar Y, Rada Espinosa ME, Aranda Gámez A, Hernández MI. Parasitosis intestinal y alternativas de disposición de excreta en municipios de alta marginalidad. Rev Cubana Salud Publica. 2008;34(2):1-4. Hallado en http:// scielo.sld.cu/scielo.php?script=sci_arttext\&p id=S0864-34662008000200009\&lng=es\&nrm= iso. Acceso el 14 de agosto de 2009.
7. Fernández-Niño J, Reyes-Harker J, MoncadaÁlvarez L, López MC, Cháves MP, Knudson $\mathrm{A}$, et al. Tendencia y prevalencia de las geohelmintiasis en La Virgen, Colombia, 19952005. Rev Salud Publica. 2007;9(2):289-96.

8. Camaño C, Albornoz F, Olarte S. Prevalencia de enteroparásitos en alumnos de la Escuela No. 82, Esquina Leales, Tucumán, Argentina. Parasitol Latinoam. 2005;60:240-1.

9. Carrizo G, Jiménez J, Quezada A, GregoriRoig P, Sánchez-Thevenet P, Fajardo M. Parásitos intestinales y estado nutricional en un grupo de niños de Comodoro Rivadavia, Chubut. Parasitol Latinoam. 2005;60:234-5.

10. Taranto N, Cajal P, de Marzi M, Fernández M, Frank M, Bru A, et al. Clinical status and parasite infection in a Wichi aboriginal community in Salta. Argentina. Trans R Soc Trop Med Hyg. 2003;97:554-8.

11. Soriano S, Manacorda A, Pierángeli $\mathrm{N}$, Navarro M, Giayetto A, Barbieri L, et al. Parasitosis intestinales y su relación con factores socioeconómicos y condiciones de hábitat en niños de Neuquén, Patagonia, Argentina. Parasitol Latinoam. 2005;60:154-61.

12. Basualdo J, Córdoba A, de Luca M, Ciarmela L, Pezzani B, Minvielle M. Intestinal parasitoses and environmental factors in a rural population of Argentina. Rev Inst Med Trop Sao Paulo. 2007;49:251-5.
13. Chiodo P, Basualdo J, Ciarmela L, Pezzani B, Apezteguía M, Minvielle M. Related factors to human toxocariasis in a rural community of Argentina. Mem Inst Oswaldo Cruz. 2006; 101:397-400.

14. Cruz M. Estudios operativos sobre el control de la teniasis/cisticercosis por Taenia solium en el Ecuador. Bol Oficina Sanit Panam. 1990;198:113-21.

15. Velasco-Castrejón O, Escobar-Gutiérrez A, Valdespino-Gómez J. Epidemiología de las helmintiasis en México. Bases para su control. México, D. F.: Instituto de Diagnóstico y Referencia Epidemiológicos; 1993. (Publicaciones Técnicas No. 24.)

16. World Health Organization. Prevention and control of intestinal parasitic infections. Report of a WHO Expert Committee. Geneva: WHO; 1987. (Technical Report Series No. 749.)

17. World Health Organization. Report of the WHO informal consultation on the use of chemotherapy for the control of the morbidity due to soil transmitted nematodes in humans. Geneva: WHO; 1996. (WHO/CTD/ SIP/96.2.)

18. Beltramino D, Lurá M, Carrera E. El tratamiento antihelmíntico selectivo frente al tratamiento masivo. Experiencia en dos comunidades hiperendémicas. Rev Panam Salud Publica. 2003;13:10-8. 
19. González García G. Programa Nacional de Tratamientos Masivos Antiparasitarios. Boletín PROAPS/REMEDIAR (Buenos Aires). 2004;2(14):1. Hallado en http://www.fe meba.org.ar/fundacion/quienessomos/Nov edades/boletines_remediar/boletinreme diar14.pdf. Acceso el 26 de octubre de 2009.

20. Ekeh H, Adeniyi J. Health education strategies for topical disease control in school children. J Trop Med Hyg. 1988;91:55-9.

21. Organización Mundial de la Salud. La iniciativa PHAST. Transformación participativa para la higiene y el saneamiento. Un nuevo enfoque para el trabajo comunitario. Ginebra: OMS; 1996. (WHO/EOS/96.11.)

22. SARAR: Self-esteem, associative strength, resourcefulness, action planning and responsibility. Washington, D.C.: World Bank; sin año. Hallado en http://go.worldbank.org/ ODBW GEOMO0. Acceso el 26 de octubre de 2009.

23. Marinof N, Pesantes M, Samanez-Carrillo C, Centurión C. Metodologías participativas en educación sanitaria. Una adaptación de PHAST para comunidades rurales andinas del Perú. Lima: Impresos Aguilar; 2001.

24. Asaolu S, Ofoezie I. The role of health education and sanitation in the control of helminth infections. Acta Trop. 2003;86:283-94.

25. Brooker S, Bethony J, Hotez P. Human hookworm infection in the 21st century. Adv Parasitol. 2004;58:197-288.

26. Dirección Provincial de Estadística de la Provincia de Buenos Aires. Censo Nacional de Población, Hogares y Viviendas 2001.
Población por Localidad. Estadísticas Sectoriales Bonaerenses (Buenos Aires). 2004;1: 1-16. Hallado en http:/ / www.ec.gba.gov.ar/ Estadistica/FTP/Censo/web1.pdf. Acceso el 26 de octubre de 2009.

27. Minvielle M, Pezanni B, Córdoba A, De Luca M, Apezteguía M, Basualdo J. Epidemiological survey of Giardia spp. and Blastocystis hominis in an Argentinean rural community. Korean J Parasitol. 2004,42:121-7.

28. Pezzani B, Minvielle M, de Luca M, Córdoba A, Apezteguía M, Basualdo J. Enterobius vermicularis infection among population of General Mansilla, Argentina. World J Gastroenterol. 2004;10:2535-9.

29. Alcheh J, Fernández G, Guarnera EA, Gutiérez N, Pizzi H, Taranto N. Guía para el manejo de las geohelmintiosis 2007. Buenos Aires: MINSAL; 2007.

30. Feldman R, Guardis M. Diagnóstico coproparasitológico. Fundamentos, normas, metodología, control de calidad. Nueva Guía Práctica. Buenos Aires: Federación Bioquímica de la Provincia de Buenos Aires; 1990.

31. Organización Mundial de la Salud. Medios auxiliares para el diagnóstico de las parasitosis intestinales. Catalogación por la Biblioteca de la Organización Mundial de la Salud. Ginebra: OMS; 1994.

32. Gungoren B, Latipour R, Regallet G, Musabaeu E. Effect of hygiene promotion on the risk of reinfection rate of intestinal parasites in children in rural Uzbekistán. Trans R Soc Trop Med Hyg. 2007,101:564-9.
33. Mohammed Mahdy AK, Lim YA, Surin J, Wan KL, Al-Mekhlafi MS. Risk factors for endemic giardiasis: highlighting the possible association of contaminated water and food. Trans R Soc Trop Med Hyg. 2008;102:465-70.

34. Leeva Uoova S, Rangsin R, Taamasri P, Naaglor T, Thathaisong U, Mungthin M. Evidence of waterborne transmission of Blastocystis hominis. Am J Trop Med Hyg. 2004;70:658-62.

35. Thompson RCA. The zoonotic significance and molecular epidemiology of Giardia and giardiasis. Vet Parasitol. 2004;126:15-35.

36. Magaró H, Echenique C, Furno G, Leiva G. Prevalencia de enteroparasitosis en la ciudad de Rosario, Argentina. Infect Microbiol Clín. 1994;7:98-102.

37. Ledesma A, Fernández G. Enteroparasitosis: factores predisponentes en población infantil de la ciudad de Resistencia, Chaco, Argentina. Corrientes: Universidad Nacional del Nordeste; 1994. (Comunicaciones Científicas y Tecnológicas 2004, Resumen M-044.)

38. Cruz Licea V, Plancarte Crespo A, Morán Álvarez C, Valencia Rojas S, Rodríguez Sánchez G, Vega Franco L. Blastocystis hominis among food vendors in Xochimilo markets. Rev Lat Microbiol. 2003,45:12-5.

Manuscrito recibido el 3 de septiembre de 2008. Aceptado para publicación, tras revisión, el 16 de diciembre de 2008.

ABSTRACT Objectives. To design, implement, and evaluate a complex plan of actions aimed at reducing parasitoses in a rural town in the province of Buenos Aires, Argentina, with the involvement of the community.

\section{Community participation in the control of intestinal parasitoses at a rural site in Argentina}

Methods. The project took place in General Mansilla, Buenos Aires province, in three stages. First, the initial epidemiological situation of intestinal parasitoses in the community was assessed by analyzing for parasites a series of stool samples and anal swabs taken from a sample of 522 individuals. Second, interventions were implemented: the pharmacological treatment of the infected individuals, and health education for the public involving local promoters. Third, the pharmacological treatment was evaluated with followup stool testing for those treated; the health education effort was evaluated through a survey and stool testing for parasites of those who had participated solely in the education.

Results. The frequency of intestinal parasitoses was $58.2 \%$; of the total, $43.9 \%$ were protozoa and $35.2 \%$, helminths. The most common pathogens were Enterobius vermicularis, Blastocystis hominis, and Giardia lamblia. The deworming treatment reduced intestinal parasitoses to $15.1 \%(P<0.001)$, and was more effective in helminths than in protozoa. Intestinal parasitoses declined following the health education intervention, in general (from $58.2 \%$ to $47.9 \%$; $P=0.019$ ), as well as for helminths (from $35.2 \%$ to $20.3 \% ; P<0.001$ ), and hygiene habits improved significantly.

Conclusions. Treatment of parasitosis and the educational intervention provided by local promoters made it possible to reduce parasitic diseases-particularly those caused by helminths-in the community under study. We recommend extending this experience to other rural communities and broadening the interventions to cutoff additional modes of transmission, such as water and food.

Key words Intestinal diseases, parasitic; health education; community health aides; Argentina.

\footnotetext{
El archivo disponible recibió correcciones según ERRATA publicada en el volumen 27 número 1 de la revista.
} 